\title{
Necrosis y perforación de un tumor de intestino delgado. Presentación de un caso.
}

\author{
Necrosis and perforation of a smallbowel tumor. About a case. \\ Oxmany Lantigua Hernández. ${ }^{1}$, Yania González Díaz. ${ }^{2}$, Lenia Bárbara Bello Ávila. ${ }^{3}$ \& \\ René Luis Escaig Olivares. ${ }^{4}$
}

\begin{abstract}
.
Introduction: The small intestine occupies $75 \%$ of the digestive tract and $90 \%$ of the absorption surface. Despite being one of the largest and most metabolically active organs, tumors that originate in it are rare for them; diagnosis is usually made at an advanced stage when the therapeutic expectations are more limited and the prognosis more uncertain. Objective: To
\end{abstract}

\section{Resumen.}

Introducción: El intestino delgado ocupa el $75 \%$ del tubo digestivo y el $90 \%$ de la superficie de absorción. A pesar de ser uno de los órganos de mayor extensión y más activos metabólicamente los tumores que en él se originan son raros. Por ello su diagnóstico suele realizarse en un estadío avanzado cuando las expectativas terapéuticas son más limitadas y el

${ }^{1}$ Hospital Dr. Mario Muñoz Monroy. Colón, Cuba, ygd75@nauta.cu (D) https://orcid.org/0000-0003-1921$\underline{9283}$

${ }^{2}$ Hospital Dr. Mario Muñoz Monroy. Colón, Cuba, Yolanda2148@nauta.cu (DD https://orcid.org/0000-00019907-8892

${ }^{3}$ Hospital Dr. Mario Muñoz Monroy. Colón, Cuba, lenia.ba@nauta.cu (iD https://orcid.org/0000-0002-2731$\underline{0741}$

${ }^{4}$ Hospital Dr. Mario Muñoz Monroy. Colón, Cuba, (D) https://orcid.org/0000-000 
present a case that undergoes surgery for an acute abdomen and in the high wake the existence of two small intestine tumors is verified Clinical Case: Of a 66-year-old male patient who comes to the body of home guards for around 3 months presents with mild colic-type abdominal pain after ingestion of meals, is admitted for study and treatment 48 hours later the pain intensifies and it is decided to intervene surgically with The presumed diagnosis of a perforated ulcer in the surgical act shows two tumors located in the jejunum and ileum and the first of them perforated. Methodology: A review of the bibliography related to small intestine tumors and gist tumors was carried out, through the analysis and synthesis of these consultations, deductions were made regarding the development and future projections of similar cases. Conclusions: small intestine neoplasms on many occasions They go unnoticed due to their low incidence and their nonspecific symptoms. It is important to increase the clinical suspicion index for these tumors in order to achieve an early diagnosis. With this, an adequate surgical resection can be achieved, which is essential to avoid complications, greater survival and quality of life.

Keywords: gastrointestinal stromal tumor. GIST. small intestine. perforation pronóstico más incierto. Objetivo: Exponer un caso que se interviene quirúrgicamente por un abdomen agudo y en el acto operatorio se comprueba la existencia de dos tumores de intestino delgado. Caso Clínico: Se expuso un paciente masculino de 66 años que acude a cuerpo de guardia porque hace alrededor de 3 meses presenta dolores abdominales tipo cólico leves después de la ingestión de comidas, se ingresa para estudio y tratamiento, 48 horas después se intensifica y se decide intervenir quirúrgicamente con el presunto diagnóstico de ulcera perforada. En el acto quirúrgico se aprecian 2 tumores localizados en yeyuno e íleon, y el primero de ellos perforados. Metodología: Se realizó una revisión de la bibliografía relacionada con las tumoraciones de intestino delgado y de los tumores de GIST. Mediante el análisis y la síntesis de estas consultas se elaboraron deducciones en relación al desarrollo y proyecciones futuras de casos semejantes. Conclusiones: Las neoplasias de intestino delgado en muchas ocasiones pasan inadvertidas por su baja incidencia y sus síntomas inespecíficos. Es importante incrementar el índice de sospecha clínica de estos tumores para así lograr un diagnóstico precoz, con este se podrá conseguir una adecuada resección quirúrgica; indispensable para evitar las complicaciones, mayor supervivencia y calidad de vida.

Palabras claves: tumor del estroma gastrointestinal. GIST. Intestino Delgado. perforación 


\section{Introducción.}

El intestino delgado ocupa el $75 \%$ del tubo digestivo y el $90 \%$ de la superficie de absorción y en su longitud que excede los 6 metros se distinguen tres porciones: duodeno, yeyuno e íleon. A pesar de ser uno de los órganos de mayor extensión y más activos metabólicamente los tumores que en él se originan son raros y los síntomas vagos e inespecíficos. Por ello su diagnóstico suele realizarse en un estadío avanzado cuando las expectativas terapéuticas son más limitadas y el pronóstico más incierto. (Pellisé M \& Castells A, 2012)

Estos tumores representan entre el 3 y el $5 \%$ de los procedentes del sistema digestivo. Dos tercios son malignos y sólo un tercio benigno. (Galindo F \& Lencinas S, 2009), representando el 1,1-2,4\% de los tumores malignos gastrointestinales. Los tumores malignos más frecuentes del intestino delgado son el adenocarcinoma, el linfoma, el sarcoma y el tumor carcinoide. De forma excepcional pueden encontrarse tumores metastásicos, en especial secundarios a melanoma, cáncer de mama o hipernefroma.

El pronóstico presenta cierta asociación con la localización anatómica, con tendencia a ser de mayor malignidad los localizados en intestino delgado frente a los localizados en el estómago. Miettinem, 2006a en un estudio de 1700 GIST gástricos, observó que el $83 \%$ eran benignos. En el duodeno, sin embargo, la mitad suelen ser malignos, en el esófago, son poco frecuentes y predominan los leiomiomas; pero más de la mitad de los GIST son malignos.

En EE. UU se diagnostican en torno a los 2000 casos nuevos de cáncer de intestino delgado por año, lo que supone una incidencia que oscila entre el 0.4 y 1 por 100000 habitantes. Tanto los tumores benignos como los malignos se presentan en torno a los 60 años de edad. (Instituto Nacional del Cáncer)

Por su parte en México la prevalencia de tumores de intestino delgado se estima en 300 casos anuales, según el reporte histopatológico de neoplasias, lo que representa el $0.32 \%$ del total de las neoplasias del tracto gastrointestinal. (Núñez Vidales, 2014) Teniendo un similar comportamiento en Cuba.

El propósito del siguiente artículo es exponer un caso que se interviene quirúrgicamente por un abdomen agudo y en el acto operatorio se comprueba la existencia de dos tumores de intestino delgado.

\section{Presentación de caso}

Paciente BHC m/b de 66 años de edad con antecedentes patológicos personales de etilismo crónico y tabaquismo. Acude a cuerpo de guardia porque hace alrededor de 3 meses presenta dolores abdominales tipo cólico leves después de la ingestión de comidas, acompañado de vómitos ocasionales y una pérdida de alrededor de 15 libras. En esta ocasión presenta dolor abdominal mantenido, de moderada intensidad en barra (epigastrio y ambos hipocondrios) acompañados de vómitos, es ingresado para estudio y tratamiento. 
Cuarenta y ocho horas después es valorado por la guardia de cirugía pues el dolor se intensifica y no alivia con la analgesia. Se observa un paciente en posición fetal, taquicárdico, ligera polipnea, abdomen excavado, que no sigue los movimientos respiratorios, dolor difuso intenso, que se exacerba con la tos y se presenta tanto a la palpación superficial como la profunda, con contractura muscular involuntaria intensa. Doloroso a la percusión y RHA abolidos.

En los complementarios se observa anemia moderada, leucocitosis a predominio de segmentados, mientras que en la gasometría arterial y el ionograma se constata una acidemia metabólica y una hipopotasemia. En el Rx de tórax se observó un neumoperitoneo y el ultrasonido abdominal muestra líquido libre en cavidad. Se decide intervenir quirúrgicamente con el presunto diagnóstico de úlcera péptica perforada.

En la laparotomía se halla una peritonitis purulenta secundaria a la perforación de un tumor de yeyuno ulcerado y con áreas de necrosis, además de un segundo tumor en íleon. Los mismos son resecados, en conjunto con una adenopatía de $2 \mathrm{~cm}$ de diámetro en el mesenterio correspondiente. Se realizó anastomosis termino-terminal y amplio lavado peritoneal y se dejan drenajes. Luego de concluido el acto quirúrgico el paciente continúa con cuidados intensivos hasta que finalmente fallece $24 \mathrm{~h}$ después a consecuencias de un shock séptico.

El estudio patológico inicial arroja un carcinoma indiferenciado multicéntrico de intestino delgado con patrón infiltrante que perfora la pared intestinal el mayor de $10 \times 5 \times 4 \mathrm{~cm}$ con presencia de necrosis y bordes quirúrgicos libres de tumor, no metástasis de ganglios linfáticos 0/3. La muestra se envía al Centro de Referencia Nacional del Hospital Hermanos Amejeiras donde se le realizan los estudios inmunohistoquímicos confirmando un tumor de GIST de intestino delgado.

\section{Metodologia.}

Se realizó una revisión de la bibliografía relacionada con las tumoraciones de intestino delgado y de los tumores de GIST. Mediante el análisis y la síntesis de estas consultas se elaboraron deducciones en relación al desarrollo y proyecciones futuras de casos semejantes. Se expuso un caso recibido en el servicio de cirugía del Hospital Docente de Colón, Matanzas, Cuba Doctor Mario Muñoz Monroy en febrero del 2019.

\section{Resultados.}

En escasas ocasiones aparecen tumores malignos a nivel del intestino delgado, aunque en los últimos años se ha incrementado su incidencia paralelamente al aumento del interés por estas neoplasias. (Martín \& Martínez, 2015) Pueden ocurrir en forma esporádica o en asociación con enfermedades genéticas como la poliposis adenomatosa familiar o el síndrome de Peutz 
Jeghers o con enfermedades inflamatorias intestinales crónicas como la enfermedad de Crohn o la celíaca. (Galindo F \& Lencinas S, 2009)

La mayoría de estos tumores son asintomáticos por largos períodos de tiempo, tienen una amplia variedad histopatológica y comportamiento clínico. Pueden debutar con síntomas inespecíficos, como manifestaciones de progresión de la enfermedad, de carácter no urgente: dolor abdominal postpandreal acompañado de náuseas y vómitos, hemorragia digestiva por lesiones ulceradas, diarrea o masa tumoral. Alteraciones del estado general como la anemia, anorexia y pérdida de peso suelen ser las únicas manifestaciones clínicas que presenten los pacientes. En urgencias se puede manifestar como obstrucción intestinal, hemorragia y perforación la cual suele verse en un número limitado de casos como ocurrió en el paciente que se reporta, diagnosticando el tumor como hallazgo en la laparotomía, coincidiendo con la literatura y documentado por Trujillo-Pérez, 2018 en un paciente operado con el diagnostico preoperatorio de tumor de colon según los estudios realizados y al intervenirlo quirúrgicamente se encontró una tumoración de yeyuno que histológicamente resultó ser un tumor del estroma gastrointestinal.

Es importante, por consiguiente, incrementar el índice de sospecha clínica para este tipo de tumores para así evitar el descubrimiento de la neoplasia en etapas avanzadas.

Existen estudios que demuestran como el retardo en el diagnóstico depende fundamentalmente de una interpretación errónea de los síntomas o de las pruebas diagnósticas por parte del clínico. Estos factores son responsables de un retraso de 8-12 meses en el diagnóstico de este tipo de tumores. En contraste, el retraso diagnóstico atribuido a la falta de reconocimiento de los síntomas por parte del paciente sólo es de dos meses. (Pellisé M \& Castells A, 2012) El tiempo promedio para el diagnóstico desde el inicio de los síntomas en pacientes con tumores de intestino delgado es de 7 meses. (Galindo F \& Lencinas S, 2009) La presentación clínica puede también variar dependiendo de la localización del tumor y del subtipo histológico. (Hatzaras, 2007) Debido a la rareza de estos tumores y a los múltiples subtipos histológicos, este tipo de neoplasias ha sido poco estudiado y su entendimiento es limitado.

Aunque no se conoce aún la causa de la incidencia tan baja de estos tumores, este hecho se atribuye a que el intestino delgado tiene una secreción de altos volúmenes de líquido alcalinos, enzimas e inmunoglobulina A, esta última por el incrementado tejido linfoideo, todos estos factores podrían contrarrestar los carcinógenos potenciales, además una menor flora bacteriana puede resultar en disminución de la conversión de los ácidos biliares en carcinógenos por microorganismos anaerobios; al contener más elementos líquidos, estos irritan menos la mucosa que los sólidos, y su rápida peristalsis favorece un período corto de exposición de la mucosa intestinal con carcinógenos y finalmente La presencia de enzimas como la benzopireno hidroxilasa, que transforma el benzopireno, un conocido carcinógeno 
presente en varios alimentos, a metabolitos menos tóxicos; está presente en concentraciones mucho más altas en el intestino delgado en comparación con el colon y estómago.(Galindo F \& Lencinas S, 2009)

Sánchez-Ramón, 2012 destacó que la presencia de grandes adenomas y la inflamación crónica de la mucosa intestinal, en particular en la enfermedad de Crohn, son factores de riesgo para desarrollar una neoplasia de intestino delgado. Así como la obesidad y el tabaquismo este último junto al alcoholismo presente entre los hábitos tóxicos del paciente operado.

No existe ningún método diagnóstico ideal para el estudio del intestino delgado. La opciones son radiológicas (tomografía computarizada, enteroclisis, tránsito intestinal y resonancia magnética) o endoscópicas (cápsula endoscópica, enteroscopia de pulsión y enterosocopia de balón simple y de doble balón)(Edge, 2010) Así afirman Rivas L \& Gómez V, 2014 quienes plantean que el intestino delgado fue un territorio inaccesible para la endoscopia durante muchos años, hace referencia los autores que la enteroscopia por pulsión permite evaluar solo el yeyuno proximal, la radiografía tiene poca especificidad y baja capacidad en la detección de lesiones mucosas planas; la enteroscopia intraoperatoria, es un procedimiento invasivo con elevada morbimortalidad. Pese a todos los medios diagnósticos que permiten el estudio de este órgano en el centro donde se recibió el paciente no consta con estas técnicas.

La enteroscopia con cápsula endoscópica (ECE) y el ultrasonido endoscópico (UE) son técnicas útiles y sencillas que permite explorar el interior del intestino delgado (ID) de forma rápida y segura, al obtener imágenes en tiempo real a su paso por el tracto digestivo y la toma de biopsia poco invasiva de forma individual. (Juanmartiñena Fernández, 2016)

Bilimoria et al, 2009 analizaron los datos clínicos de tumores malignos de intestino delgado de la Base de Datos Nacional de Cáncer (NCDB) y de la Base de Datos de Vigilancia Epidemiológica (SEER) de Estados Unidos de los últimos 20 años, encontrando que los adenocarcinomas son la neoplasia más común en duodeno, los tumores neuroendocrinos se encuentran con mayor frecuencia en el íleon, los linfomas en el yeyuno, mientras que los sarcomas se desarrollan de manera indistinta en cualquier parte del intestino.

En el caso que reportamos la biopsia concluyó que se trataba de un tumor de GIST multicéntrico. En esta denominación conocida por sus siglas en inglés (gastrointestinal stromal tumor) e introducido según Trujillo-Pérez, 2018 por Mazur y Clark en 1983, se incluyen los tumores del estroma o mesénquima del tubo digestivo y estructuras contiguas, que pueden surgir en cualquier punto, desde el estómago hasta el año.

El GIST es un tumor subepitelial que anteriormente fue clasificado como mesenquimal (nomenclatura previa), como el leiomioma, el tumor de la vaina de los nervios, el 
schwannoma y el lipoma; este concepto es ampliamente aceptado y ha permitido diferenciarlo por su origen, comportamiento y tratamiento.

Estos tumores pueden ser benignos o comportarse como maligno, algunas características de su malignización son: afección a la mucosa o epitelio, es decir, ulceración, hemorragia y presencia de coágulos. (Mederos CON, 2020)

Los tumores del estroma gastrointestinal, pertenecen a la familia de los "sarcomas de tejidos blandos", son las neoplasias no epiteliales más frecuentes del tracto gastrointestinal y derivan de precursores de las células intersticiales de Cajal presentes en el plexo mesentérico; cuya función es la de servir de marcapaso del sistema gastrointestinal regulando la peristálsis o en células troncales relacionadas. Estas células generan contracciones del músculo liso y recibirían señales de neuronas autonómicas que difunden a las células musculares. (Miettinen M \&Lasota J, 2006)

Aunque el GIST continúa siendo un raro tipo de cáncer, cuanto más se estudia, menos infrecuente parece su incidencia. Su incidencia en las series reportadas es de 4-10 casos/millón de habitantes/año. Es la neoplasia mesenquimática más común del tracto digestivo; sin embargo, su frecuencia representa solo un 0,1 a $3 \%$ de las neoplasias gastrointestinales. Teniendo una prevalencia mayor, debido el curso clínico largo de la enfermedad (10-15 años). (Luna Gozá, 2011)

Su ubicación más frecuente es a nivel del estómago (60-70\%), seguido por su localización en intestino delgado (30\%), colorrectal (5\%) y sólo 2-4\% es encontrado en el esófago (muy infrecuente en el apéndice, y otros sitios). La edad promedio de presentación es entre los 50 y 70 años, sin diferencia en la incidencia entre géneros. (Soler-Vaillant, 2018).

El tamaño oscila entre 0,3 a $40 \mathrm{~cm}$ pudiendo tener crecimiento tanto hacia la luz intestinal como hacia afuera. (Galindo F \& Lencinas S, 2009)

Debido a que su crecimiento suele ser extraluminal y su consistencia blanda, los tumores no se presentan en forma de obstrucción del tránsito intestinal (incidencia menor al 10\%), por lo que son asintomáticos al inicio de la enfermedad. (Pedroso Rodríguez, 2012) Los síntomas aparecen en los tumores mayores de $5 \mathrm{~cm}$.

Frecuentemente se ulceran, que puede llegar a la perforación y se presentan como un abdomen agudo por peritonitis; (Roccatagliata N, 2020; Villasanti N, 2019) así fue observado en el paciente que se recibió en nuestro hospital que presentó una perforación en el tumor ulcerado que además tenía áreas de necrosis.

La presencia de tumores primarios múltiples en un paciente constituye una condición clínica actualmente diagnosticada con mayor frecuencia. Por lo tanto, se denomina tumores primarios múltiples a las neoplasias que se presentan en un sujeto de forma simultánea o 
sucesiva siempre que cada tumor tenga definido un patrón de malignidad; que no constituya metástasis de otro; que cada uno tenga una histología diferente; si ambos son similares dentro del mismo órgano, se debe demostrar que no hay ninguna conexión entre ellos; cada tumor debe seguir su historia natural; pueden tener evoluciones independientes, si el diagnóstico es simultáneo o dentro de los primeros 6 meses se denominan sincrónicos, si su diagnóstico es sucesivo se llaman metacrónicos.

Poco más del 37\% resultan ser tumores multicéntricos en el mismo órgano; en el caso que exponemos precisamente su peculiaridad está dada porque siendo un tumor infrecuente en cuanto a localización, tipo histológico y forma de presentación como una perforación se hallan durante el acto quirúrgico dos focos de tumoración comprobándose mediante la biopsia ser una neoplasia multicéntrica.

La multifocalidad se define como la presencia de 2 o más focos tumorales en un mismo cuadrante y a menos de $5 \mathrm{~cm}$ del foco primario, y la multicentricidad es la presencia de $2 \mathrm{o}$ más focos tumorales en sitios distintos a más de $5 \mathrm{~cm}$ del foco primario. Sin embargo, los tumores primarios sincrónicos son escasamente frecuentes. (Adad, 2011)

Los GIST tanto benignos como malignos pueden ser submucosos, subserosos o intraluminales observándose como una masa nítida con atenuación homogénea y a veces calcificaciones. El cirujano familiarizado con los distintos tipos de patología del intestino delgado puede hacer el diagnóstico macroscópico en la mayor parte de los casos.

El diagnóstico histológico de estos tumores requiere de técnicas inmunohistoquímicas mediante la expresión del factor de crecimiento de la tirosina kinasa como el CD117 que es siempre positiva. Otro marcador no constante es el CD 34 (34 al 70\%) (González Cámpora, 2017; Martín Bourricaudy, 2015)

La histología muestra que estos tumores se presentan con células fusiformes en la mayor parte de los casos 70 a $86 \%$ los que tienen mejor expectativa de vida, tipo epiteliode 5 a $20 \%$ y mixto $10 \%$ o menos. En intestino delgado son más frecuentes los fusocelulares mientras los epiteliodes se dan más en estómago. Así lo corroboran Landell Cruz J \& Martínez Velázquez C, 2016 y Verdecia Cañizares C, 2017. El reconocimiento de estos tumores GIST al igual que las células intersticiales de Cajal es porque ambos expresan la proteína KIT (tirosina-kinasa). Se conoce la mutación del gen responsable de la expresión de la proteína KIT (cromosoma 4q11-q12). La mutación produce una proteína anormal (KIT fosforilada) que provoca proliferación celular e inhibe la apoptosis. Los verdaderos leiomiomas y schwannoma no dan positiva la reacción con CD117.

El tratamiento de elección es el quirúrgico con la resección más completa posible, los malignos deben ser siempre resecados con suficiente margen $(>6 \mathrm{~cm}$.) proximal y distal. En 
aquellos donde no es posible resecar la lesión el tratamiento paliativo está indicado mediante el uso del mesilato de imatinib, que actúa por medio de la inhibición específica de la enzima tirosina quinasa importante en la patogenia de estos tumores, así se detiene su proliferación y se induce la apoptosis, dosificado con una duración de un año es capaz de prolongar la supervivencia libre de recaída en los tumores localizados y con diámetro de $3 \mathrm{~cm}$ o más con una resección macroscópica completa, según reportan Ferrer Robaina H, 2017 y ValdésPeregrina, 2018 at el respectivamente.

La resección por vía laparoscópica es una variante del tratamiento quirúrgico, la técnica es segura y tiene buen pronóstico oncológico, pero según afirman Martín Bourricaudy \& Martínez Alfonso, 2015 es fundamental evitar la ruptura del tumor, pues conlleva a un pronóstico semejante al alcanzado si no fuera posible resecar completamente la lesión.

La biopsia no es recomendable en el preoperatorio cuando se considera que son tumores operables con posibilidades de resección. El diagnóstico definitivo lo da el estudio histopatológico de la pieza quirúrgica incluyendo reacciones específicas de inmunohistoquímica. Estos tumores dan su diseminación por vía hemática por lo que la búsqueda de adenopatías no siempre está justificada y la lifadenectomía no es necesaria ya que solo de manera excepcional y rara vez en formas avanzadas metastizan a ganglios linfáticos. (Pedroso Rodríguez, 2012)

El pronóstico se relaciona con la extensión de la enfermedad (Chok, 2015) y el índice mitótico desafortunadamente, como se mencionó anteriormente, la rareza de estas lesiones y la inespecificidad de su comportamiento clínico entorpecen el diagnóstico precoz. El sitio donde se encuentra el tumor es importante, pues los intestinales tienden a ser más agresivos, en relación a las localizaciones gástricas. Otros factores serían los subtipos histológicos, el grado de pleomorfismo celular y la edad del paciente. De esta forma la ausencia de perforación y un índice mitótico inferior a 5/50 se considera de valor pronóstico favorable. (Pérez Tauriaux, 2015)

En general, el pronóstico de estos tumores es pobre, se estima que la sobrevida relativa a 5 años es menor al 30\%. Según plantea Wu, 2006 el 50\% de los tumores resecados con criterio curativo a los 5 años han fallecido. Jiménez Cubedo, 2017 consideran que de un 40 a un 60 $\%$ de los pacientes desarrollarán una recidiva en un plazo de dos años, ya sea como metástasis o recurrencia local tras la resección primaria de estos tumores. 


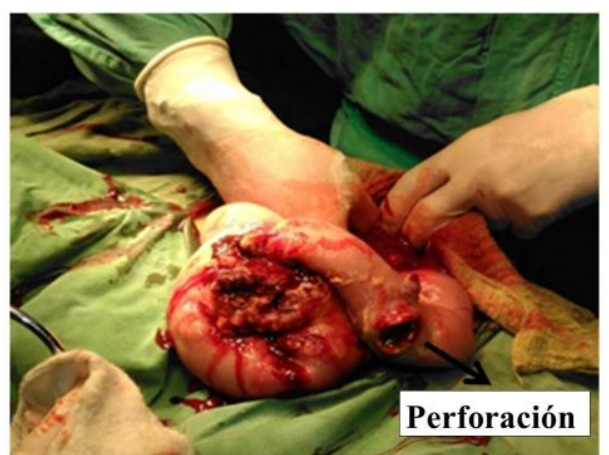

Figura 1 Obsérvese un primer tumor en yeyuno con áreas de necrosis y la perforación.

Fuente: Foto tomada durante el acto quirúrgico

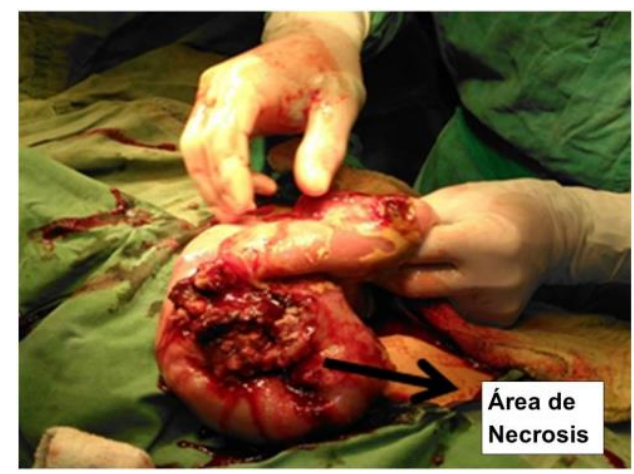

Figura 2 Tumor de Yeyuno. Obsérvese el área de necrosis

Fuente: Foto tomada durante el acto quirúrgico

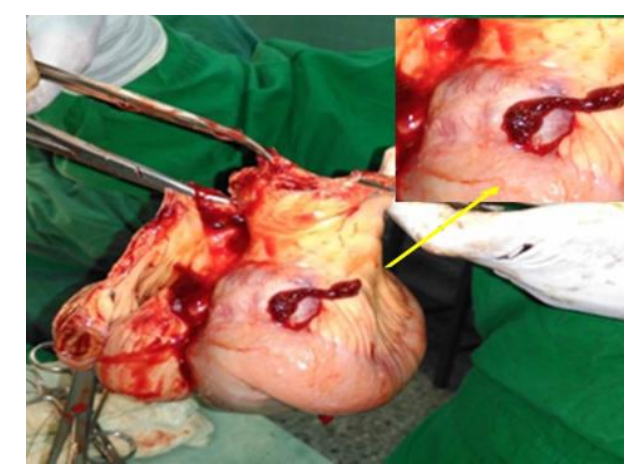

Figura 3 Obsérvese un segundo tumor en íleon Fuente: Foto tomada durante el acto quirúrgico

\section{Conclusiones.}


- Las neoplasias de intestino delgado en muchas ocasiones pasan inadvertidas por su baja incidencia y sus síntomas inespecíficos.

- Es importante incrementar el índice de sospecha clínica de estos tumores para así lograr un diagnóstico precoz.

- La resección quirúrgica oportuna es indispensable para evitar las complicaciones y mayor supervivencia con calidad de vida.

\section{Referencias bibliográficas.}

Adad S.J, Canteras Raposo Camara C.A, Mota Pereira J, do Carmo J. Jr, Rua Micheletti J.A.M. (2011) Adenocarcinoma primário multicêntrico com 12 focos. Relato de caso e revisão da literatura. Rev Bras Colo-proctol, 31, pp. 205-209

Bilimoria K, Bentrem D, Wayne J, et al. (2009) Small bowel cancer in the United States: changes in epidemiology, treatment, and survival over the last 20 years. Ann Surg.; 249:63---71.

Chok AY, Goh BK, Koh YX, Lye WK, Allen JC Jr, Quek R, et al. (2015) Validation of the MSKCC gastrointestinal stromal tumor nomogram and comparison with other prognostication systems: Single-institution experience with 289 patients. Ann Surg Oncol. 22 (11): 3597-3605.

Edge, SB, Byrd, DR, Compton, CC et al., eds. AJCC (American Joint Committee on Cancer) (2010) Cancer staging Manual, 7. a edición, Springer, New York:181, 285,175.

Ferrer Robaina H, Rodríguez Cruz Y, Mesa Izquierdo O, Zayas Díaz L, Blanco Amaro E.(2017) Tumor de estroma gastrointestinal en intestino delgado. Rev cubana Cir [Internet]. [citado 9 oct 2020];56(2). Disponible en: http://revistaamc.sld.cu/8122017

Galindo F y Lencinas S (2009) Tumores del intestino delgado. Cirugía Digestiva II-261, pág. 1-17. Disponible en: www.sacd.org.ar

González Cámpora R, Ramos Asensio R, Vallejo Benítez A, Marcilla Plaza D, Biscuola M, Martínez Marín V, et al. (2017) Tumores del estroma gastrointestinal: breve actualización y consenso de la SEAP-SEOM sobre diagnóstico patológico y molecular. Rev Esp Patol [Internet]. [citado 9 oct 2020]; 50(2): [aprox. 9 p.]. Disponible

en:

http://web.a.ebscohost.com/ehost/pdfviewer/pdfviewer?vid=0\&sid=1a4b01b-31984676-99e7-e3e16beb086c\%40sessionmgr4008

Instituto Nacional del Cáncer [Internet]. Bethesda, MD: NIH; [citado 16 Abr 2020]. Cáncer de intestino delgado-Versión para profesionales de salud. Disponible en: https://medlineplus.gov/spanish/intestinalcancer.html 
Jiménez Cubedo E, Lucena de la Poza JL, Artés Caselles M, Sánchez Turrión V. (2017) Metástasis hepatodiafragmática de tumores del estroma gastrointestinal duodenal. Rev Chil Cir [Internet]. [citado 9 Feb 2018];69(4):[aprox. 12 p.]. Disponible en: https://scielo.conicyt.cl/scielo.php?script=sci_arttext\&pid=S071840262017000400002\&lng=es\&nrm=iso\&tlng=es

Juanmartiñena Fernández JF, FernándezUrién I, Saldaña Dueñas C, Elosua González A, Borda Martín A, Vila Costas JJ. (2016) Detección de lesiones fuera del intestino delgado con cápsula endoscópica en pacientes con hemorragia digestiva oculta. Anales Sis San Navarra [Internet]. Ago [citado 15 sep 2020]; 39(2): [aprox. 3 p.]. Disponible en: http://scielo.isciii.es/scielo.php?script=sci_arttext\&pid=S1137$\underline{66272016000200015 \& \ln g=e}$

Landell Cruz J, Martínez Velázquez C. (2016) Tumor del estroma gastrointestinal del estómago. Rev electrón [Internet]. [citado 26 sep 2020];41(8):[aprox. 12 p.]. Disponible en: http://www.revzoilomarinello.sld.cu/index.php/zmv/article/view/76010

Luna Gozá MM, Portales Pérez R, Echevarría Hernández F, Valdés JL, Satorre Roche J (2011) Tumor del estroma gastrointestinal del intestino delgado. Revista Cubana de Cirugía;50(4):590-596 Disponible en http://scielo.sld.cu

Martín Bourricaudy N \& Martínez Alfonso MA. (2015) Resección laparoscópica de tumor del estroma gástrico. Rev Cubana Cir [Internet]. Jun [citado 20 sep 2020];54(2):[aprox. $\quad 6 \quad$ p.]Disponible en: http://scielo.sld.cu/scielo.php?script=sci_arttext\&pid=S0034$\underline{74932015000200008 \& \operatorname{lng}=\mathrm{es}}$

Martínez Sanz N, Ruiz Marín M, González Valverde FM, Sánchez Cifuentes A, Fernández López AJ, Ródenas Moncada FJ y Marín Blázquez AA. (2016) Rev esp enfeRm dig. Vol. 108, N. ${ }^{\circ}$, pp. $432-433$

Mederos CON, Barrera OJC, López SA, Mederos TON, González LJ. (2020) Sangrado digestivo bajo masivo por tumor de GIST en un divertículo de Meckel. Cir Gen.; 42(1): 45-49. doi: 10.35366/92711

Miettinem M, Majidi M, Lasota J. (2002) Pathology and diagnostic criteria of gastrointestinal stromal tumors (GISTs): a review. Eur J Cancer. 38 Suppl. 5: S39-S51.

Miettinem M \& Lasota J. (2006a) Gastrointestinal stromaltumors: pathology and prognosis at differentsites. Semin Diagn Pathol. 23:70-83. 
Miettinem M \& Lasota J. (2006b) Gastrointestinal stromal tumors: review on morphology, molecular pathology, prognosis, and differential diagnosis. : Arch Pathol Lab Med.130:1466-78

Núñez Vidales R, José Luis Martínez-Ordaz y Alicia Estrada Castellanos.(2014) Prevalencia de tumores malignos primarios y metástasicos de intestino delgado. Cir Gen 36(4):214-217. Disponible en: www.elservier.es/cirujanogeneral

Pellisé M, Castells A. (2012) Sección 4. Intestino delgado y colon. Capítulo 30. Tumores del intestino delgado. En: Montoro MA, García Pagán JC, editores. Gastroenterología y Hepatología. Problemas comunes en la práctica clínica [Internet]. 2da ed. Madrid: Jarpyo Editores; [citado 10 Feb 2020]. Disponible en: https://www.aegastro.es/sites/default/files/archivos/ayudaspracticas/30_Tumores_de 1_intestino_delgado.pdf

Pedroso Rodríguez M, Mora de la Paz T, Hernández Meza F, Castillo Arias R. (2012) Tumor de GIST: Presentación de dos casos clínicos. Revista Médica de Costa Rica y Centroamérica LXIX (603) 373-377

Pérez Tauriaux O, González Bernardo R. (2015) Tumor del estroma gastrointestinal de localización gástrica. MEDISAN [Internet]. Feb [citado 26 Ene 2020]; 19(2): [aprox. 5 p.]. Disponible en: http://scielo.sld.cu/scielo.php?script=sci_arttext\&pid=S1029$\underline{30192015000200015 \& \operatorname{lng}=\mathrm{es}}$

Rivas L \& Gómez V. (2014) Enteroscopía como método diagnóstico para neoplasias de intestino delgado. Crea Ciencia [Internet]. Jun [citado 15 sep 2020]; 9(1): [aprox. 4 p.]. Disponible en: http://web.b.ebscohost.com/ehost/pdfviewer/pdfviewer?vid=1\&sid=a3ff26b7-85634adaa02e-31cab79b86e9\%40pdc-v-sessmgr01

Roccatagliata N, Nebil Larrañaga N, Espil G, Salgado R, Hernández Pinzón J, Yepes Hernández C, Cajamarca J, Kozima S. (2020) Masa a nivel de íleon distal, Vol. 50 N² - 2020 (Jun-Sept) 895 Lecturas Acta Gastroenterol Latinoam; 50(2):140-143

Sánchez-Ramón A, Cerino-Palomino V \& Medina-Franco H. (2012) Tumores de intestino delgado: experiencia en el Instituto Nacional de Ciencias Médicas y Nutrición “'Salvador Zubirán'’ Revista de Gastroenterología de México. 77(4):181---185

Soler-Vaillant R, Mederos-Curbelo ON. (2018) Cirugía. Tomo VI: Cirugía del tubo digestivo. Parte 11. Afecciones quirúrgicas del intestino delgado. RodríguezRodríguez I, Martín-González MA. Capítulo 144. Tumores estromales de intestino delgado y del colon. La Habana, Cuba: Editorial Ciencias Médicas: pp. 293-306. ISBN 978-959-313-106-3 
Valdés Peregrina EN, Hernández González M, León Pacheco Ode, Mendoza Ramírez S. (2018) Tumor del estroma extra-gastrointestinal. Informe de un caso de tumor primario en epiplón. Rev Méd Hosp Gen Méx [Internet]. [citado 9 oct 2020];81(4):[aprox. 5 p.]. Disponible en: https://reader.elsevier.com/reader/

Verdecia Cañizares C, Villamil Martínez R, Montero Reyes I, Pineda Fernández D. (2017) Tumor estromal gastrointestinal. Rev Cubana Pediatr [Internet]. Mar [citado 26 Ene 2020]; 89 (1): [aprox. $6 \quad$ p.]. Disponible en: http://scielo.sld.cu/scielo.php?script=sci_arttext\&pid=S003475312017000100007\&lng=es

Villasanti N, Gerson D, González A, Ojeda LM, Segovia L, Herald, Noguera Arzamendia JC, Riquelme Recalde EK. (2019)Tratamiento del tumor del estroma gastrointestinal como hallazgo casual. A propósito de un caso. Hospital de Clínicas, Paraguay Cir. Urug. Vol. 3, No Esp. dic. DOI: 10.31837/cir.urug/3.esp.1

Wu TJ, Lee LY, Yeh CN, Wu PY, Chao TC, Hwang TL, Jan YY, Chen MF (2006) Surgical treatment and prognostic analysis for gastrointestinal stromal tumors GISTs) of the small intestine: before the era of imatinib mesylate. BMC Gastroenterol. Oct 24; 6:29.

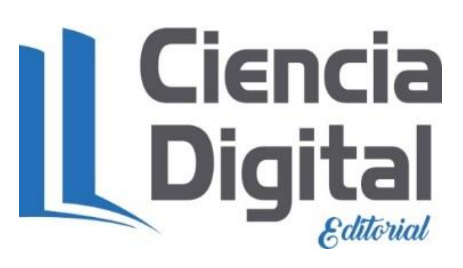




\section{PARA CITAR EL ARTÍCULO INDEXADO.}

González Díaz, Y., Lantigua Hernández, O., Bello Ávila, L. B., \& Escaig Olivares, R. L. (2021). Necrosis y perforación de un tumor de intestino delgado. Presentación de un caso . Anatomía Digital, 4(1), 40-54. https://doi.org/10.33262/anatomiadigital.v4i1.1480

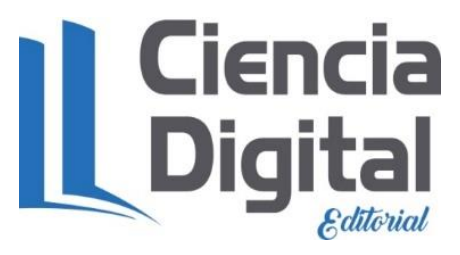

El artículo que se publica es de exclusiva responsabilidad de los autores y no necesariamente reflejan el pensamiento de la Revista Anatomía Digital.

El artículo queda en propiedad de la revista y, por tanto, su publicación parcial y/o total en otro medio tiene que ser autorizado por el director de la Revista Anatomía Digital.
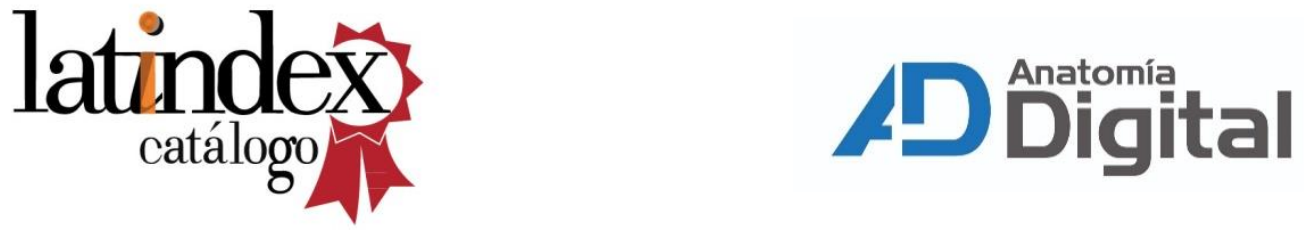